\title{
A NECESSARY AND SUFFICIENT CONDITION FOR SIMULTANEOUS DIAGONALIZATION OF TWO HERMITIAN MATRICES AND ITS APPLICATION
}

\author{
by YIK-HOI AU-YEUNG $\dagger$
}

(Received 4 February, 1969)

1. Introduction and statement of the theorems. We denote by $F$ the field $R$ of real numbers, the field $C$ of complex numbers, or the skew field $H$ of real quaternions, and by $F^{n}$ an $n$ dimensional left vector space over $F$. If $A$ is a matrix with elements in $F$, we denote by $A^{*}$ its conjugate transpose. In all three cases of $F$, an $n \times n$ matrix $A$ is said to be hermitian if $A=A^{*}$, and we say that two $n \times n$ hermitian matrices $A$ and $B$ with elements in $F$ can be diagonalized simultaneously if there exists a non singular matrix $U$ with elements in $F$ such that $U A U^{*}$ and $U B U^{*}$ are diagonal matrices. We shall regard a vector $u \in F^{n}$ as a $1 \times n$ matrix and identify a $1 \times 1$ matrix with its single element, and we shall denote by diag $\left\{A_{1}, \ldots, A_{m}\right\}$ a diagonal block matrix with the square matrices $A_{1}, \ldots, A_{m}$ lying on its diagonal.

Let $A=\operatorname{diag}\left\{A_{1}, \ldots, A_{m}\right\}$ and $B=\operatorname{diag}\left\{B_{1}, \ldots, B_{m}\right\}$ be any two hermitian block matrices such that, for each $k=1, \ldots, m, A_{k}$ and $B_{k}$ are of the same size. Then it is obvious that, if each pair $A_{k}$ and $B_{k}$ can be diagonalized simultaneously, so also can the pair $A$ and $B$. Whether the converse is true or not is not at all obvious. In this note the author gives a simple proof of the converse (Theorem 2) by first proving the following theorem on a necessary and sufficient condition for simultaneous diagonalization of two hermitian matrices.

THeOrem 1. Let $A$ and $B$ be two $n \times n$ hermitian matrices with elements in $F$. Then $A$ and $B$ can be diagonalized simultaneously if and only if there exists a basis $\left\{u_{1}, \ldots, u_{n}\right\}$ of $F^{n}$ such that, for each $i=1, \ldots, n$, the two vectors $u_{i} A$ and $u_{i} B$ are linearly dependent over $R$.

THEOREM 2. Let $A=\operatorname{diag}\left\{A_{1}, \ldots, A_{m}\right\}$ and $B=\operatorname{diag}\left\{B_{1}, \ldots, B_{m}\right\}$ be two hermitian diagonal block matrices with elements in $F$ such that, for each $k=1, \ldots, m, A_{k}$ and $B_{k}$ are of the same size. If $A$ and $B$ can be diagonalized simultaneously, then so also can the pair $A_{k}$ and $B_{k}$ for each $k$.

A theorem similar to Theorem 1, on the simultaneous diagonalization of two nondegenerate symmetric bilinear forms over a field of characteristic not equal to 2 , has been established by M. J. Wonenburger [3, Theorem 1, p. 617].

2. Proof of Theorem 1. Suppose that $A$ and $B$ can be diagonalized simultaneously. Then there exists a basis $\left\{u_{1}, \ldots, u_{n}\right\}$ such that $u_{i} A u_{j}^{*}=u_{i} B u_{j}^{*}=0$ for all $i \neq j(i, j=1, \ldots, n)$. Now, for each fixed $i$, if $u_{i} A u_{i}^{*}=u_{i} B u_{i}^{*}=0$, then $u_{i} A=0=u_{i} B$, while if $u_{i} A u_{i}^{*}$ and $u_{i} B u_{i}^{*}$ are not both zero, then $\left(u_{i} B u_{i}^{*}\right) u_{i} A-\left(u_{i} A u_{i}^{*}\right) u_{i} B=0$. Hence in both cases $u_{i} A$ and $u_{i} B$ are linearly dependent over $R$.

† The author wishes to thank Professor Y. C. Wong for his advice during the preparation of this note. 
To prove the sufficiency of the condition, suppose that there exists a basis $X=\left\{u_{1}, \ldots, u_{n}\right\}$ of $F^{n}$ such that, for each $i=1, \ldots, n, u_{i} A$ and $u_{i} B$ are linearly dependent over $R$. Then, for each $i$, there exist $\alpha_{i}, \beta_{i} \in R$, not both zero, such that

$$
\alpha_{i} u_{i} A+\beta_{i} u_{i} B=0 .
$$

Now in the set $X=\left\{u_{1}, \ldots, u_{n}\right\}$ we define a relation $\sim$ by setting $u_{i} \sim u_{j}$ if $\alpha_{i} \beta_{j}-\alpha_{j} \beta_{i}=0$. Obviously this is an equivalence relation. Let

$$
X=X_{1} \cup X_{2} \cup \ldots \cup X_{m}
$$

be the partition defined by this relation. Then, for each $k=1, \ldots, m$, there exist $a_{\mathrm{k}}, b_{\mathrm{k}} \in R$, not both zero, such that

$$
\begin{aligned}
a_{k} u A+b_{k} u B=0, & \text { for all } u \in X_{k} ; \\
a_{k} b_{l}-a_{l} b_{k} \neq 0, \text { for all } & k \neq l(k, l=1, \ldots, m) .
\end{aligned}
$$

From these two properties and $\left(u A v^{*}\right)^{*}=v A u^{*}$, it follows immediately that

$$
u A v^{*}=u B v^{*}=0 \text {, for all } u \in X_{k} \text { and } v \in X_{l} \text { with } k \neq l \text {. }
$$

Without loss of generality we may assume that $u_{1}, \ldots, u_{n_{1}} \in X_{1}, u_{n_{1}+1}, \ldots, u_{n_{1}+n_{2}} \in X_{2}, \ldots$, $u_{n_{1}+n_{2}+\cdots+n_{m-1}+1}, \ldots, u_{n} \in X_{m}$. Let $U$ be the matrix whose elements in ith row are the components of $u_{i}$. Then $U$ is non singular and, by (3), we have

$$
\begin{aligned}
& U A U^{*}=\operatorname{diag}\left\{A_{1}, \ldots, A_{m}\right\}, \\
& U B U^{*}=\operatorname{diag}\left\{B_{1}, \ldots, B_{m}\right\},
\end{aligned}
$$

where $A_{k}$ and $B_{k}$ are hermitian matrices of size $n_{k}$ and, by (1), we have

Hence

$$
a_{k} u A v+b_{k} u B v=0 \text { for all } u, v \in X_{k} .
$$

$$
a_{k} A_{k}+b_{k} B_{k}=0 \text { for each } k=1, \ldots, m .
$$

Since any hermitian matrix can be diagonalized (for $F=R$ or $C$, this is well-known; for $F=H$, see [1] or [2]) and $a_{k}, b_{k}$ are not both zero, $A_{k}$ and $B_{k}$ can be diagonalized simultaneously for each $k$. Hence $A$ and $B$ can be diagonalized simultaneously.

3. Proof of Theorem 2. It suffices to prove the theorem for $m=2$. Let $A=\operatorname{diag}\left\{A_{1}, A_{2}\right\}$ and $B=\operatorname{diag}\left\{B_{1}, B_{2}\right\}$, where $A_{1}$ and $B_{1}$ are of size $n_{1}$ and $A_{2}$ and $B_{2}$ are of size $n_{2}$, and let $n=n_{1}+n_{2}$. If $A$ and $B$ can be diagonalized simultaneously, then, by Theorem 1, there exists a basis $\left\{u_{1}, \ldots, u_{n}\right\}$ of $F^{n}$ such that, for each $i=1, \ldots, n, u_{i} A$ and $u_{i} B$ are linearly dependent over $R$.

Let $u_{i}=\left(x_{i}, y_{i}\right)$, where $x_{i} \in F^{n_{1}}$ and $y_{i} \in F^{n_{2}}$. Then $\left(x_{i} A_{1}, y_{i} A_{2}\right)$ and $\left(x_{i} B_{1}, y_{i} B_{2}\right)$ are linearly dependent over $R$ for each $i$. Hence $x_{i} A_{1}$ and $x_{i} B_{1}$ are linearly dependent over $R$ for each $i$. Since $\left\{u_{1}, \ldots, u_{n}\right\}$ is a basis of $F^{n}$, there exists $\left\{x_{i_{1}}, \ldots, x_{i_{1}}\right\}$ which forms a basis of $F^{n_{1}}$. By Theorem $1, A_{1}$ and $B_{1}$ can be diagonalized simultaneously. Similarly, $A_{2}$ and $B_{2}$ can be diagonalized simultaneously. This completes the proof. 


\section{REFERENCES} $1-14$.

1. J. Radon, Lineare Scharen orthogonaler Matrizen, Abh. Math. Sem. Univ. Hamburg 1 (1922),

2. H. C. Lee, Eigenvalues and canonical forms of matrices with 'quaternion coefficients, Proc. Roy. Irish Acad. Sect. A 52 (1949), 253-260.

3. M. J. Wonenburger, Simultaneous diagonalization of symmetric bilinear ,forms, $J$. $\mid$ Math. Mech. 15 (1966), 617-622.

UNIVERSITY OF HONG KONG 